\title{
Caracterização de carbonatos bimodais integrando Pressão Capilar por Mercúrio e Ressonância Magnética Nuclear
}

${ }^{*}$ Frederico Bastos Schuab ${ }^{1,2}$; Alessandra Winter ${ }^{1}$, Osvair Vidal Trevisan" ${ }^{1}$

${ }^{1}$ Universidade Estadual de Campinas, ${ }^{2}$ Petrobras

Copyright 2014, SBGt - Sociedade Brasileira de Geofísica

Este texto foi preparado para a apresentação no VI Simpósio Brasileiro de Geofísica, Porto Alegre, 14 a 16 de outubro de 2014. Seu conteúdo foi revisado pelo Comitê Técnico do VI SimBGf, mas não necessariamente representa a opinião da SBGf ou de seus associados. É proibida a reproducão total ou parcial deste material para propósitos comerciais sem prévia autorização da SBG

\section{Resumo}

Esse trabalho apresenta a caracterização de rochas carbonáticas com distribuição de porosidade bimodal utilizando a técnica de Ressonância Magnética Nuclear (RMN). Foram integrados resultados de análises laboratoriais de petrofísica básica e especial de 49 plugues de um reservatório de petróleo para determinação da porosidade, permeabilidade e saturação de água através da RMN. As rochas apresentam textura grainstone, com altas permeabilidades e porosidades, porém com microporosidade também alta, reveladas pelas análises de Pressão Capilar por Intrusão de Mercúrio $(\mathrm{PcHg})$ e pela distribuição do tempo de relaxação transversal $\left(T_{2}\right)$. A bimodalidade do sistema poroso com micro e macroporos bem definidos, porém conectados, exerce controles diferentes sobre o fluxo de fluidos (permeabilidade) e sobre a distribuição de saturações, sendo recomendável, como conclui esse trabalho, a utilização de cortes de $\mathrm{T}_{2}$ diferentes para cálculo da saturação de água irredutível e da permeabilidade absoluta a partir do RMN.

\section{Introdução}

A importância dos reservatórios carbonáticos na indústria do petróleo já é conhecida, principalmente devido aos grandes campos do Oriente Médio e, mais recentemente, às descobertas do Pré-Sal.

Muitos trabalhos mostram que o conhecimento do sistema poroso dessas rochas é fundamental para a caracterização das formações carbonáticas. Nesse contexto, a textura das rochas tem papel relevante.

De acordo com o trabalho original de Dunham (1962), as rochas carbonáticas são classificadas segundo a sua textura deposicional. Quando reconhecível em lâminas petrográficas, são separadas segundo a abundância de grãos (Figura 1). Nessa classificação, os grainstones são rochas compostas apenas por grãos e não há matriz fina, ou lama carbonática. Por isso, são também chamados carbonatos granulares em muitas publicações.

Os carbonatos de textura grainstone apresentam predominância de grãos de oólitos e oncolitos, como mostra a Figura 2. Segundo Terra et al. (2010), esses grãos são esféricos a subelípticos, originados por acreção físico-química e organo-sedimentar, respectivamente, com tamanhos entre $0,2 \mathrm{~mm}$ (oólitos) e $5 \mathrm{~mm}$ (oncolitos) de diâmetro. Dada a esfericidade e arredondamento desses grãos, a analogia pode ser feita com uma caixa de bolas de gude, sendo essas os grãos e o espaço entre elas a porosidade. No entanto, como mostram Cantrell e Hagerty (1999), esses grãos apresentam grande quantidade de microporosidade intragranular.

Essa grande quantidade de microporosidade afeta significativamente os resultados das análises, principalmente a determinação da saturação e da permeabilidade, como é discutido nesse trabalho.

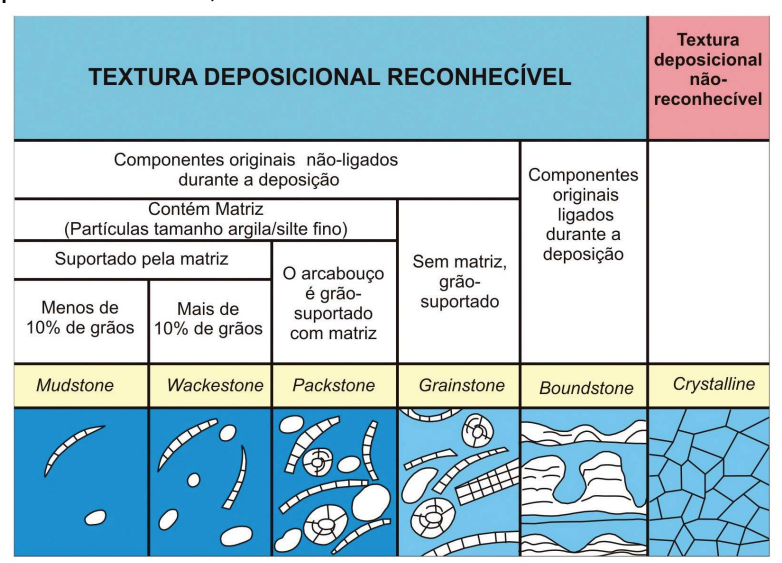

Figura 1 - Classificação para rochas carbonáticas baseada na textura (adaptado de Dunham, 1962 apud Terra et al., 2010).

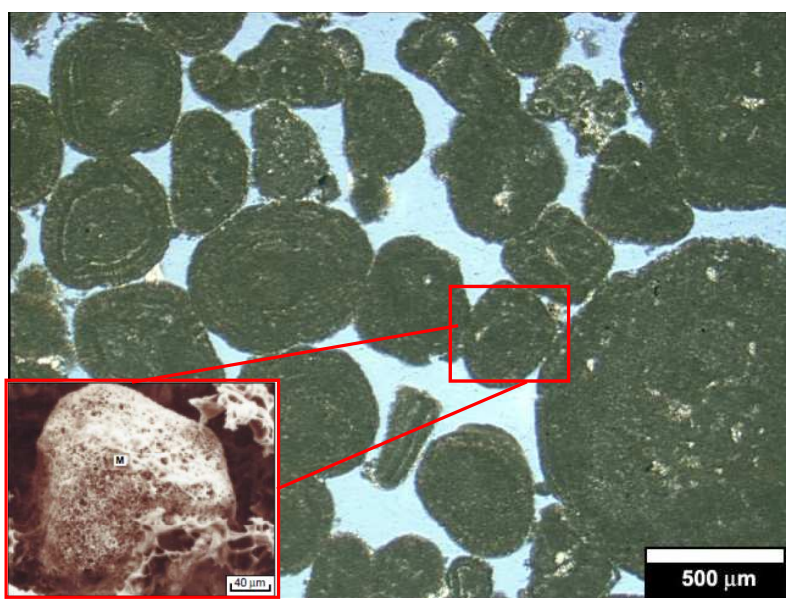

Figura 2 - Foto de lâmina petrográfica mostrando a textura grainstone e os grãos de oólitos e oncolitos da amostra 227. Detalhe destacando um grão microporoso (adaptado de Cantrell e Hagerty, 1999). 


\section{Metodologia}

Para esse trabalho, foram selecionadas 49 amostras cilíndricas de $3,7 \mathrm{~cm}$ de diâmetro e comprimento médio de $3,94 \mathrm{~cm}$ pertencentes a um intervalo de 33 metros de testemunhos de um reservatório carbonático de petróleo. Todas as amostras foram submetidas a análises de porosidade e permeabilidade pela PETROBRAS com porosímetro e permeabilímetro a gás e pressão de confinamento superior a 500 psi.

Do montante inicial foram selecionados os resultados de ensaios de Pressão Capilar por Intrusão de Mercúrio $(\mathrm{PcHg})$ realizados pela PETROBRAS em 41 plugues e os resultados dos ensaios de Ressonância Magnética Nuclear (RMN), realizados no CEPETRO/UNICAMP, de 8 plugues cedidos pela PETROBRAS. A Tabela 1 apresenta a relação desses ensaios e os valores de porosidade e permeabilidade de cada amostra.

\section{Pressão Capilar por Intrusão de Mercúrio $(\mathrm{PcHg})$}

Os ensaios de $\mathrm{PcHg}$ foram realizados pela PETROBRAS em 40 amostras conforme a Tabela 1.

Nesta análise, o mercúrio é injetado sob pressões escalonadas de 1 até 60.000 psia e volumes conhecidos nas amostras de rocha. Como o Mercúrio não molha a superfície da rocha é possível penetrar gargantas de poros com raios de até 0,001 micrômetros. Sabendo-se a pressão de injeção e o volume de mercúrio intrudido, é possível obter uma curva de pressão capilar no sistema $\mathrm{Hg}$-Ar. Assumindo que as gargantas se comportam como um modelo de tubo capilar, é possível determinar o raio de garganta de poro (rgp) que controla o fluxo de mercúrio a partir da equação de Laplace, sendo inversamente proporcional a pressão de injeção.

Onde:

$$
P_{c}=\frac{2 \sigma \cdot \cos \theta}{r} \times C
$$

$\mathrm{P}_{\mathrm{C}}=$ Pressão capilar em psi

$\sigma=$ tensão superficial em dina/cm $(\mathrm{Hg}=480 \mathrm{dina} / \mathrm{cm})$;

$\theta=$ ângulo de contato em graus $\left(\mathrm{Hg} / \mathrm{ar} /\right.$ sólido $\left.=140^{\circ}\right)$;

$r=$ raio de garganta de poros em angstrons;

$\mathrm{C}=$ fator de conversão de unidades.

\section{Ressonância Magnética Nuclear (RMN)}

As medidas do tempo de relaxação transversal $\left(T_{2}\right)$ foram realizadas em 8 amostras a $35^{\circ} \mathrm{C}$ utilizando 0 equipamento Modelo GeoSpec 2-53/Maran DRX HF (2,2 $\mathrm{MHz}$ ) fabricado pela Oxford Instruments, Reino Unido, equipado com sonda de $53 \mathrm{~mm}$. A seqüência de pulso utilizada para a medição do $T_{2}$ foi a CPMG (Carr-PurcellMeiboom-Gill). As distribuições de $T_{2}$ foram obtidas através do cálculo da transformada inversa de Laplace das medidas de CPMG, utilizando-se o programa WinDXP versão.1.8.1.0. Os parâmetros de medição foram: espaçamento entre Ecos (TE) de 0,2 ms e razão Sinal/Ruído (SNR) superior a 100.

As análises de RMN foram realizadas nas 8 amostras, primeiramente $100 \%$ saturadas com salmoura de $200 \mathrm{kppm}$ e, em seguida, na condição de saturação de água irredutível (Swirr), obtida após a centrifugação das amostras em uma Ultra-Centrífuga (URC-628 UltraRock Centrifugue - Coretest Systems, INC) até a velocidade de 7000 rpm.

Tabela 1 - Relação de amostras e ensaios para os 49 plugues de 1,5".

\begin{tabular}{|c|c|c|c|c|}
\hline AMOSTRA & DIREÇÃO & POROS & PERME & ENSAIOS \\
\hline 171 & $\mathrm{H}$ & 19,46 & 2 & $\mathrm{PcHg}$ \\
\hline 174 & $\mathrm{H}$ & 19,53 & 1,25 & $\mathrm{PcHg}$ \\
\hline 176 & $\mathrm{H}$ & 19,27 & 0,78 & RMN/Cent \\
\hline 180 & $\mathrm{H}$ & 26 & 126,92 & $\mathrm{PcHg}$ \\
\hline 182 & $\mathrm{H}$ & 22,84 & 398,22 & $\mathrm{RMN} /$ Cent/PcHg \\
\hline 184 & $\mathrm{H}$ & 22,93 & 42,26 & $\mathrm{PcHg}$ \\
\hline 185 & $\mathrm{H}$ & 21,8 & 84,63 & $\mathrm{PcHg}$ \\
\hline 189 & $\mathrm{H}$ & 24,74 & 285,24 & $\mathrm{PcHg}$ \\
\hline 191 & $\mathrm{H}$ & 25,79 & 106,21 & $\mathrm{PcHg}$ \\
\hline 193 & $\mathrm{H}$ & 23,36 & 628,55 & sem condições \\
\hline 195 & $\mathrm{H}$ & 29,19 & 191,7 & $\mathrm{PcHg}$ \\
\hline 198 & $\mathrm{H}$ & 30,4 & 136,98 & $\mathrm{PcHg}$ \\
\hline 199 & $\mathrm{H}$ & 30,92 & 118,98 & $\mathrm{PcHg}$ \\
\hline 200 & $\mathrm{H}$ & 27,91 & 67,66 & $\mathrm{PcHg}$ \\
\hline 202 & $\mathrm{H}$ & 28,11 & 70,47 & $\mathrm{PcHg}$ \\
\hline 203 & $\mathrm{H}$ & 27,69 & 181,31 & $\mathrm{PcHg}$ \\
\hline 206 & $\mathrm{H}$ & 20,27 & 114,78 & $\mathrm{PcHg}$ \\
\hline 208 & $\mathrm{H}$ & 22,77 & 842,75 & $\mathrm{PcHg}$ \\
\hline 211 & $\mathrm{~V}$ & 24,52 & 968,9 & RMN/Cent \\
\hline 212 & $\mathrm{H}$ & 24,22 & 498,71 & $\mathrm{PcHg}$ \\
\hline 215 & $\mathrm{H}$ & 23,14 & 800,21 & $\mathrm{PcHg}$ \\
\hline 221 & $\mathrm{H}$ & 21,42 & 357,84 & $\mathrm{PcHg}$ \\
\hline 222 & $\mathrm{H}$ & 26,35 & 285,14 & $\mathrm{PcHg}$ \\
\hline 225 & $\mathrm{H}$ & 23,41 & 364,96 & $\mathrm{PcHg}$ \\
\hline 227 & $\mathrm{H}$ & 27,08 & 479,77 & $\mathrm{PcHg}$ \\
\hline 228 & V & 26,49 & 571,49 & RMN/Cent \\
\hline 229 & $\mathrm{H}$ & 25,24 & 876,88 & $\mathrm{PcHg}$ \\
\hline 230 & $\mathrm{H}$ & 28,86 & 784,98 & $\mathrm{PcHg}$ \\
\hline 231 & $\mathrm{H}$ & 25,52 & 529,89 & $\mathrm{PcHg}$ \\
\hline 233 & $\mathrm{H}$ & 21,97 & 260,99 & $\mathrm{PcHg}$ \\
\hline 235 & $\mathrm{H}$ & 24,86 & 248,19 & $\mathrm{PcHg}$ \\
\hline 236 & $\mathrm{H}$ & 24,53 & 272,96 & $\mathrm{PcHg}$ \\
\hline 237 & $\mathrm{H}$ & 25,73 & 269,28 & $\mathrm{PcHg}$ \\
\hline 240 & $\mathrm{H}$ & 25,34 & 213,68 & $\mathrm{PcHg}$ \\
\hline 242 & $\mathrm{H}$ & 23,88 & 138,75 & $\mathrm{PcHg}$ \\
\hline 243 & $\mathrm{H}$ & 25,64 & 342,44 & RMN/Cent \\
\hline 244 & $\mathrm{H}$ & 24,4 & 152,2 & $\mathrm{PcHg}$ \\
\hline 247 & $\mathrm{H}$ & 25,25 & 391,35 & $\mathrm{PcHg}$ \\
\hline 248 & $\mathrm{H}$ & 22,88 & 272,15 & $\mathrm{PcHg}$ \\
\hline 258 & $\mathrm{H}$ & 28,08 & 385,15 & RMN/Cent \\
\hline 263 & $\mathrm{H}$ & 28,34 & 447 & $\mathrm{PcHg}$ \\
\hline 264 & $\mathrm{H}$ & 31,8 & 223,94 & $\mathrm{PcHg}$ \\
\hline 266 & $\mathrm{H}$ & 32,38 & 654,05 & RMN/Cent \\
\hline 270 & $\mathrm{H}$ & 30,6 & 354,04 & $\mathrm{PcHg}$ \\
\hline 272 & $\mathrm{H}$ & 29,93 & 408,31 & $\mathrm{PcHg}$ \\
\hline 276 & $\mathrm{H}$ & 29,47 & 1198,55 & $\mathrm{PcHg}$ \\
\hline 277 & $\mathrm{H}$ & 29,32 & 1561,97 & RMN/Cent \\
\hline 287 & $\mathrm{H}$ & 26,52 & 4,3 & $\mathrm{PcHg}$ \\
\hline 293 & $\mathrm{H}$ & 21,98 & 1,03 & $\mathrm{PcHg}$ \\
\hline$H / \mathrm{V}$ & \multicolumn{4}{|c|}{ Horizontal / Vertical } \\
\hline Cent & \multicolumn{4}{|c|}{ Centrífuga Ar-Água } \\
\hline RMN & \multicolumn{4}{|c|}{ Ressonância Magnética Nuclear } \\
\hline $\mathrm{PcHg}$ & \multicolumn{4}{|c|}{ Pressão Capilar por Intrusão de Mercứio } \\
\hline
\end{tabular}




\section{Resultados e Discussões}

Os resultados das análises de petrofísica básica revelaram amostras com altas porosidades e com permeabilidades bastante variáveis. A Tabela 2 apresenta as médias e a variabilidade dos valores.

Tabela 2 - Resultado das análises de petrofísica básica das 49 amostras.

\begin{tabular}{|c|c|c|}
\cline { 2 - 3 } \multicolumn{1}{c|}{} & Porosidade (\%) & Permeabilidade (mD) \\
\hline Média & 25,55 & 361,63 \\
\hline Mínimo & 19,27 & 0,78 \\
\hline Máximo & 32,38 & 1561,97 \\
\hline Desvio Padrão & 3,30 & 329,00 \\
\hline
\end{tabular}

Quando visualizadas em um gráfico de correlação, a variação da permeabilidade é confirmada e resulta em uma baixa correlação com a porosidade (Figura 3).

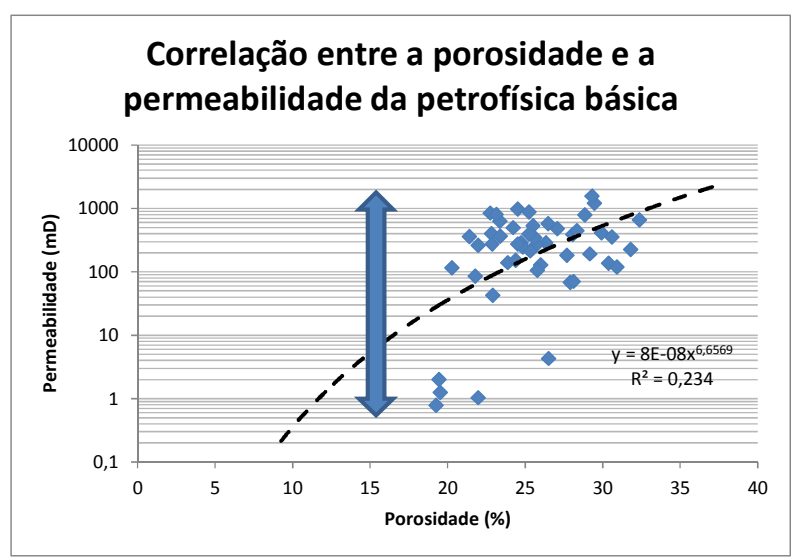

Figura 3 - Baixa correlação entre a porosidade e a permeabilidade, que apresenta grande variabilidade, indicada pela seta azul.

Nesta figura a presença de microporosidade é sugerida devido às amostras apresentarem alta porosidade mesmo para baixos valores de permeabilidade.

\section{$\mathrm{PcHg}$}

Como pode ser verificado na Figura 4, praticamente todas as amostras apresentam bimodalidade da distribuição de raios de garganta de poros (rgp). Considerando a proporcionalidade entre poros e gargantas - poros grandes são conectados por gargantas grandes e poros pequenos por pequenas gargantas podemos extrapolar a afirmação para distribuição bimodal de porosidade.

Essa distribuição de porosidade, mostra claramente que a rocha é composta por poros conectados por gargantas de raio inferiores a 0,5 micrômetros e superiores a 5 micrômetros. A mesoporosidade pode ser interpretada como a conexão entre os dois sistemas. Sabendo-se tratar de textura grainstone, fica evidente que a porosidade entre os grãos (intergranular) é o macroporo com rgp $>5$ micras e que o microporo (rgp $<0,5$ micras) só podem estar associados ao grão, ou seja intragranular (dentro dos grãos). Cantrell e Hagerty (1999) discutiram a ocorrência dessa microporosidade em rochas semelhantes na Formação Arab-D. Rahman et al. (2012) tratam da classificação de microporosidade em carbonatos apresentando uma breve revisão.

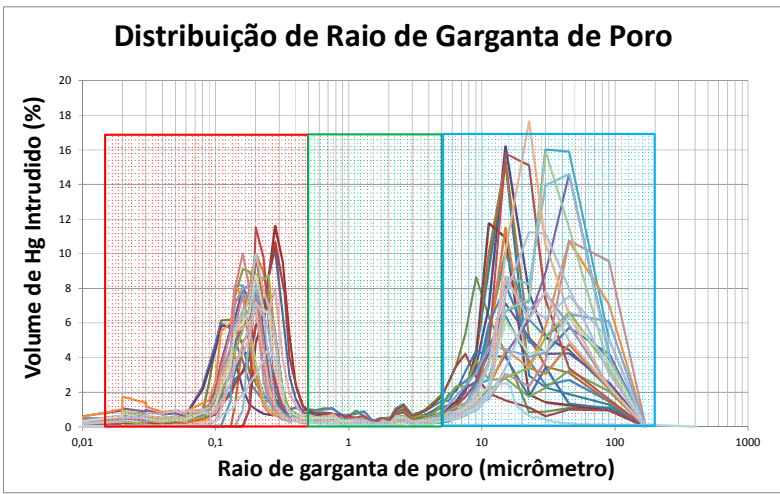

Figura 4 - Distribuição de raios de garganta de poros das 41 amostras. As áreas vermelha, verde e azul indicam as zonas micro, meso e macroporosas, respectivamente.

Como já foi mostrado na Figura 3 , os altos valores de porosidade para amostras com baixa permeabilidade e a dispersão apresentada no gráfico de correlação, sugerem a existência de poros que pouco contribuem para a permeabilidade. No entanto, quando são correlacionados os valores de macroporosidade, correspondente ao volume de $\mathrm{Hg}$ intrudido nos raios de garganta de poros maiores que 5 micras, com a permeabilidade da petrofísica básica verifica-se um bom ajuste, confirmando que a principal contribuição para o fluxo é dos macroporos (Figura 5).

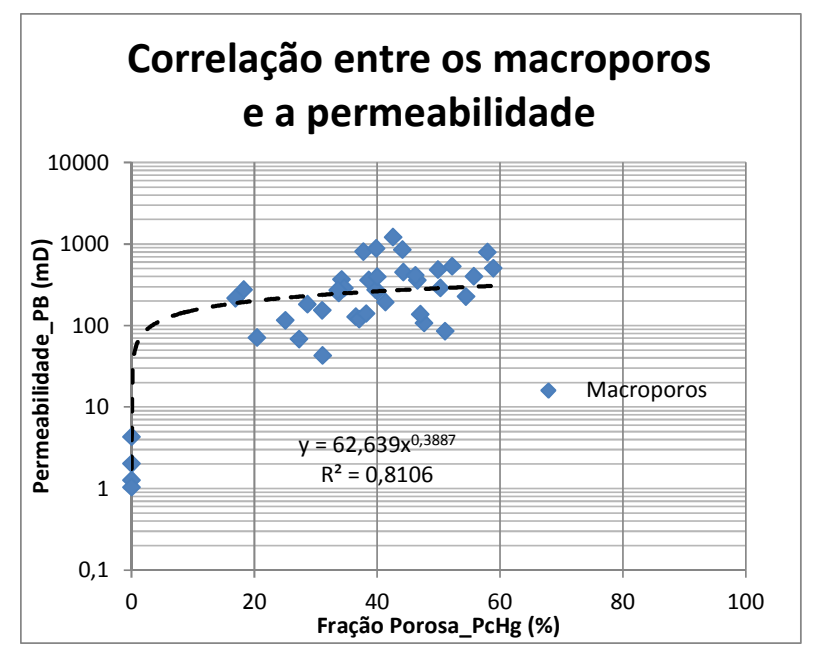

Figura 5 - Correlação entre a macroporosidade e a permeabilidade da petrofísica básica com $R^{2}=81 \%$. 


\section{RMN}

A Figura 6 apresenta a distribuição de $T_{2}$ para todas as amostras $100 \%$ saturadas. A distribuição do tempo de decaimento mostra o mesmo comportamento (bimodal) da distribuição de rgp, permitindo concluir que existe proporcionalidade entre tamanho de poros e de gargantas

Nesta condição de saturação, predominam o efeito de relaxação superficial, descartando-se os efeitos difusivos e "bulk", como descrito em Coates et al. (1999).

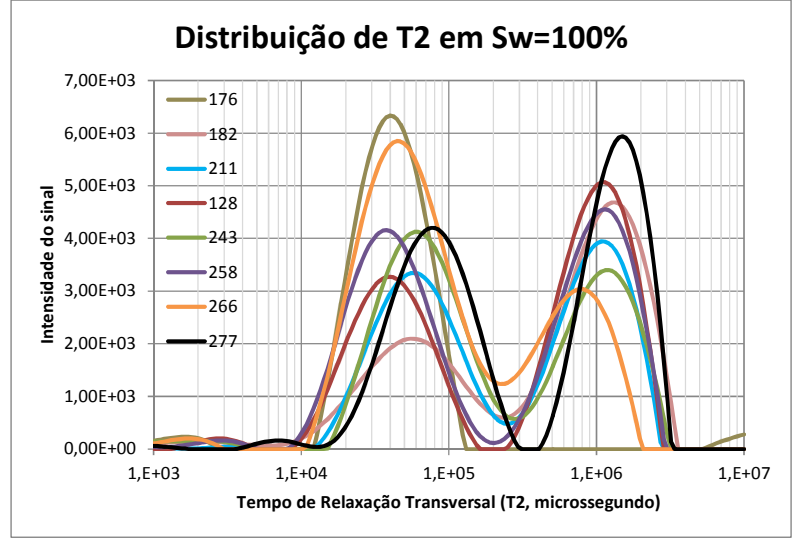

Figura 6 - Distribuição de $T_{2}$ com saturação total em salmoura 200kppm. A exceção da amostra 176, todas as demais apresentam comportamento bimodal.

A porosidade foi obtida diretamente a partir da amplitude inicial do sinal de RMN das amostras $100 \%$ saturadas e a comparação com os valores obtidos pelo porosímetro é mostrada na Figura 7.

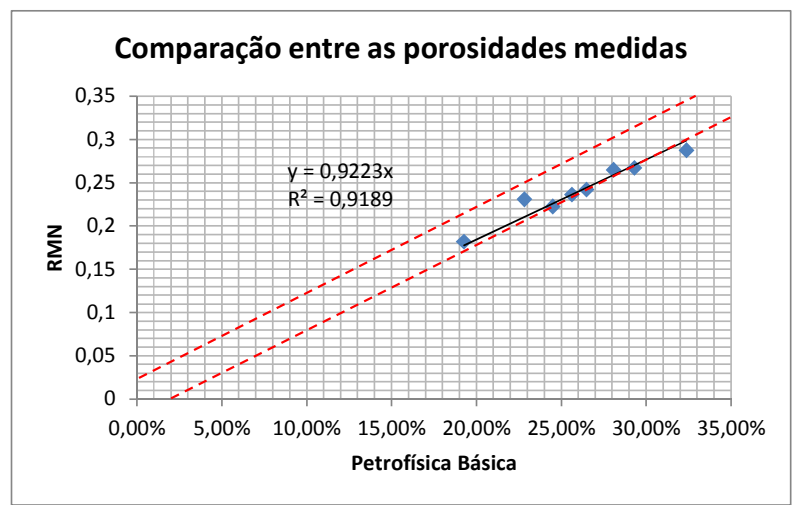

Figura 7 - Comparação entre a porosidade determinada pelo $R M N$ e a da Petrofísica Básica. As linhas pontilhadas correspondem a 2 unidades de porosidade (p.u.).

A porosidade obtida pelo RMN foi mais baixa que a da petrofísica básica, com desvio de até $2,6 \%$, o que pode estar relacionado à saturação incompleta dos microporos.

Para a determinação da permeabilidade e da saturação de água, foi necessária a interpretação das curvas de distribuição de $T_{2}$ através da definição de um corte de tempo, chamado $\mathrm{T}_{2 \text { cutoff, }}$ que tem por objetivo separar a área do espectro correspondente a fluidos capilares (ou imóveis) da área correspondente aos fluidos móveis.

Como o tamanho dos poros é o controle primário na determinação da quantidade de fluido capaz de se mover e como o espectro de $T_{2}$ reflete o tamanho dos poros, a área com tempos menores que o de corte representa a saturação de água irredutível.

Já a permeabilidade, conforme o trabalho de Timur (1968), é quase sempre baseada na relação da porosidade total ou área de fluido livre (maiores tempos) pela área de fluidos capilares. Como estes últimos estão relacionados a poros pequenos ou ao estrangulamento desses, entende-se que os poros maiores, ou o centro desses, são os maiores contribuintes para a permeabilidade. Assim, o corte definido para determinação da saturação de água irredutível é aplicado também na determinação da permeabilidade, como é apresentado na literatura.

A definição do corte $\mathrm{T}_{2 \text { cutoff }}$ descrita por Coates et al. (1999) é baseada na distribuição de $T_{2}$ da amostra 100\% saturada e na condição de Swirr da mesma amostra. Com base nessa metodologia, dispôs-se no mesmo gráfico as curvas incrementais (distribuição) e as curvas acumuladas de $T_{2}$, nas duas condições de saturação. $A$ partir do ponto final da curva acumulada de Swirr traçouse uma reta paralela ao eixo das abscissas. O ponto de intersecção dessa reta com a curva acumulada 100\% saturada definiu o valor do $T_{2}$ de corte $\left(T_{2 \text { cutoff }}\right)$. A Figura 8 mostra a aplicação dessa metodologia na amostra 182.

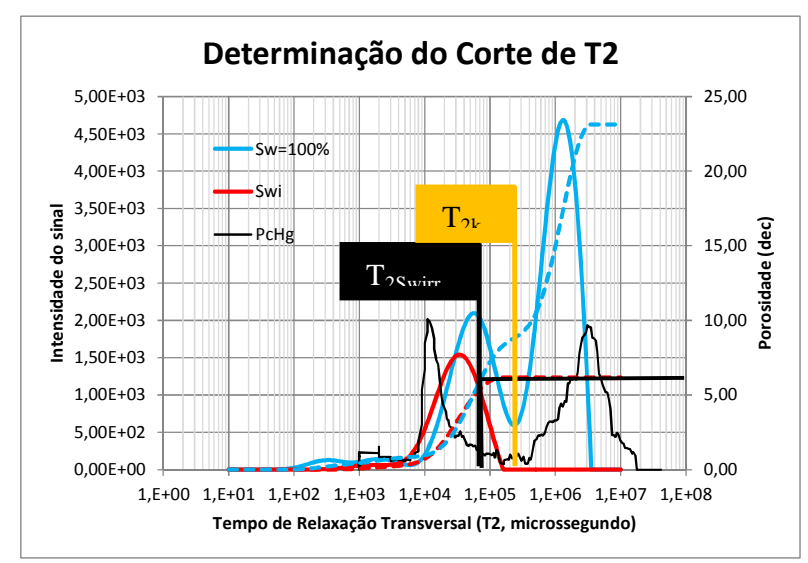

Figura 8 - Determinação do corte de $T_{2}\left(T_{2 \text { Swirr }}\right.$ com base nas curvas $100 \%$ saturadas e Swirr da amostra 182 para determinação da saturação de água irredutível. Corte definido pelas linhas pretas em $65 \mathrm{~ms}$. Também é apresentado o corte de $T_{2 K}(250 \mathrm{~ms})$ que está relacionado à separação entre as modas micro e macroporosas. A curva preta mostra a distribuição de rgp da mesma amostra, a única com os dois ensaios.

Como pode ser notado, o corte definido para determinação da saturação de água irredutível, denominado nesse trabalho de $\mathrm{T}_{2 \text { Swirr, ocorre dentro da }}$ parcela microporosa, sugerindo que, a depender da molhabilidade, o óleo pode atingir poros de escala micro. 
No entanto, como já foi visto nos resultados de $\mathrm{PcHg}$, a macroporosidade é a principal responsável pela permeabilidade da rocha, fato esse confirmado ao se comparar a macroporosidade obtida pelo RMN com a permeabilidade da petrofísica básica, como mostra a Figura 9.

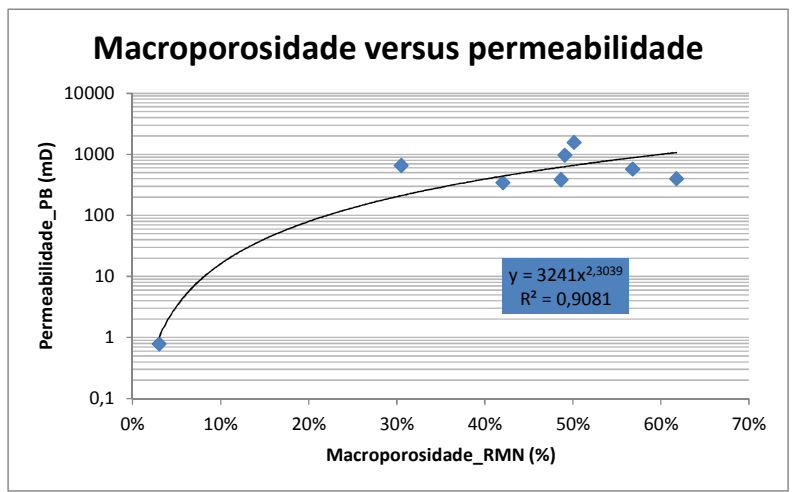

Figura 9 - Correlação entre a macroporosidade obtida pela razão entre as áreas a direita e a esquerda do corte de $T_{2 K}$ e a permeabilidade da petrofísica básica.

Com base na correlação entre as duas medidas de permeabilidade, conforme vista na Fig.9, foi definido outro corte de $T_{2}$, chamado de $T_{2 k}$, para determinação da macroporosidade e consequentemente da permeabilidade. A expressão final da permeabilidade é:

$$
K_{\text {NMR }}=3241 \times M A C R O^{2,3039}
$$

onde Macro se refere ao valor da macroporosidade da formação.

O formato da equação é semelhante às encontradas nos principais modelos citados na literatura, como Timur (1968) e Coates et al. (1999), entre outros, utilizados até hoje.

Todos os resultados da interpretação dos ensaios de PcHg e do RMN são apresentados na Tabela 3.

\section{Conclusões}

Os resultados das análises de $\mathrm{PcHg}$ e de $\mathrm{RMN}$ com amostras $100 \%$ saturadas mostram correspondências, indicando uma rocha com distribuição bimodal de porosidade bem marcada. Esse comportamento está intimamente relacionado à textura da rocha (grainstone).

A correspondência entre os resultados das análises de $\mathrm{PcHg}$ e RMN é importante para a validação da proporcionalidade entre os raios de garganta e porosidade e, consequentemente, da metodologia. Com base nisso, conclui-se que não há ocorrência significativa de poros grandes conectados por pequenas gargantas.

A grande quantidade de poros que pouco contribuem para a permeabilidade (microporos) numa rocha bimodal torna a correlação entre a porosidade e a permeabilidade difusas. Quando a correlação é feita com a macroporosidade é verificada uma boa correlação, evidenciando que esta fração porosa é a principal responsável pelo fluxo.

Essa conclusão permitiu a determinação de mais um corte de $T_{2}\left(T_{2 K}\right)$ separando macro e microporos para determinação da permeabilidade, além do tradicional corte de água irredutível ( $\left.\mathrm{T}_{2 \text { 2Swirr }}\right)$.

Esse estudo mostra que, a depender da molhabilidade da formação e da distribuição de porosidades, a utilização de um segundo corte na interpretação de resultados do RMN para determinação da permeabilidade pode ser uma boa prática, evitando os ajustes matemáticos dos coeficientes das tradicionais equações de permeabilidade, sem significado físico.

\section{Agradecimentos}

A Petrobras por ter cedido os dados e amostras e financiado o presente estudo.

\section{Referências}

DUNHAM R. J., 1962. Classification of Carbonate rocks According to Depositional Texture. AAPG, Memoir 1, Houston, Texas.

TERRA G. J. S., SPADINI A. R., FRANÇA A. B., SOMBRA C. L., ZAMBONATO E. E., JUSCHAKS L. C. S., ARIENTI L. M., ERTHAL M. M., BLAUTH M., FRANCO M. P., MATSUDA N. S., SILVA N. G. C., MORETTI JUNIOR P. A., D'AVILA R. S. F., SOUZA R. S., TONIETTO S. N., ANJOS S. M. C., CAMPINHO V. S., WINTER W. R., 2010. Classificação de Rochas Carbonáticas Aplicável às Bacias Sedimentares Brasileiras. Boletim de Geociências da Petrobras, Rio de Janeiro, V. 18, n. 1, p. 9-29.

TIMUR A., 1968. An Investigation of Permeability, Porosity \& Residual Water Saturation Relationships for Sandstone Reservoirs. The Log Analyst.

COATES G. R., XIAO L., PRAMMER M. G., 1999. NMR Logging Principles and Applications. Halliburton Energy Services, Houston, 235p.

CANTRELL D. L., HAGERTY R. M., 1999. Microporosity in Arab Formations Carbonates, Saudi Arabia. GeoArabia, Vol. 4, №. 2.

RAHMAN M. H., PIERSON B. J., YUSOFF W. I. W., 2012. Classification of Microporosity: Examples from Miocene Carbonate Reservois of Central Luconia, Offshore Sarawak, Malaysia. IPTC, Bangkok, Thailand, 79 fevereiro.

ROMERO P., GOMEZ N., 2004. Method for Characterization of Rock Quality Based on WinlandPittman and Timur-Coates Equations Applied to NMR Laboratory Data. SPWLA 45th Annual Logging Symposium, June 6-9. 
Tabela 3 - Resultados finais da caracterização.

\begin{tabular}{|c|c|c|c|c|c|c|c|c|c|c|c|c|}
\hline $\begin{array}{c}\text { Amostra } \\
\mathrm{s}\end{array}$ & $\begin{array}{l}\text { Poros } \\
\text { (\%) }\end{array}$ & $\begin{array}{l}\text { Perme } \\
\text { (mD) }\end{array}$ & $\begin{array}{c}\text { Micro_PcHg } \\
(\%) \\
\text { rgp }<0,5 \mu \mathrm{m}\end{array}$ & \begin{tabular}{|c|} 
Meso_PcHg \\
$(\%)$ \\
$0,5<$ rgp $<5 \mu \mathrm{m}$
\end{tabular} & \begin{tabular}{|c|} 
Micro_PcHg \\
$(\%)$ \\
rgp $>5 \mu \mathrm{m}$
\end{tabular} & $\begin{array}{l}\mathrm{T}_{2 \mathrm{Swirr}} \\
\text { (ms) }\end{array}$ & $\begin{array}{l}\mathrm{T}_{2 \mathrm{~K}} \\
(\mathrm{~ms})\end{array}$ & $\begin{array}{c}\text { Micro_- } \\
\text { RMN } \\
(\%)\end{array}$ & $\begin{array}{c}\text { Macro__ } \\
\text { RMN } \\
(\%)\end{array}$ & $\begin{array}{c}\text { Poros__ } \\
\text { RMN } \\
(\%)\end{array}$ & $\begin{array}{l}\mathrm{K}_{\mathrm{RMN}} \\
(\mathrm{mD})\end{array}$ & $\begin{array}{l}\text { Swi } \\
\text { RMN } \\
(\%)\end{array}$ \\
\hline 171 & 19,46 & 2 & 94,66 & 5,33 & 0,00 & & & & & & & \\
\hline 174 & 19,53 & 1,25 & 95,95 & 4,05 & 0,00 & & & & & & & \\
\hline 176 & 19,27 & 0,78 & & & & 38,00 & 100,00 & $95,93 \%$ & $3,03 \%$ & $18,12 \%$ & 1,03 & $48,83 \%$ \\
\hline 180 & 26 & 126,92 & 47,29 & 16,17 & 36,54 & & & & & & & \\
\hline 182 & 22,84 & 398,22 & 36,63 & 7,58 & 55,79 & 65,00 & 250,00 & $38,19 \%$ & $61,81 \%$ & $23,03 \%$ & 1069,66 & $24,40 \%$ \\
\hline 184 & 22,93 & 42,26 & 51,95 & 16,93 & 31,13 & & & & & & & \\
\hline 185 & 21,8 & 84,63 & 41,40 & 7,53 & 51,08 & & & & & & & \\
\hline 189 & 24,74 & 285,24 & 45,35 & 4,35 & 50,30 & & & & & & & \\
\hline 191 & 25,79 & 106,21 & 43,64 & 8,75 & 47,62 & & & & & & & \\
\hline 193 & 23,36 & 628,55 & & & & & & & & & & \\
\hline 195 & 29,19 & 191,7 & 51,92 & 6,69 & 41,39 & & & & & & & \\
\hline 198 & 30,4 & 136,98 & 45,93 & 7,02 & 47,05 & & & & & & & \\
\hline 199 & 30,92 & 118,98 & 51,73 & 11,17 & 37,11 & & & & & & & \\
\hline 200 & 27,91 & 67,66 & 60,94 & 11,77 & 27,29 & & & & & & & \\
\hline 202 & 28,11 & 70,47 & 64,60 & 14,97 & 20,44 & & & & & & & \\
\hline 203 & 27,69 & 181,31 & 63,37 & 7,97 & 28,66 & & & & & & & \\
\hline 206 & 20,27 & 114,78 & 64,43 & 10,53 & 25,04 & & & & & & & \\
\hline 208 & 22,77 & 842,75 & 50,22 & 5,65 & 44,13 & & & & & & & \\
\hline 211 & 24,52 & 968,9 & & & & 70,00 & 250,00 & $50,87 \%$ & $49,13 \%$ & $22,22 \%$ & 630,26 & $32,11 \%$ \\
\hline 212 & 24,22 & 498,71 & 33,93 & 7,13 & 58,94 & & & & & & & \\
\hline 215 & 23,14 & 800,21 & 57,52 & 4,69 & 37,80 & & & & & & & \\
\hline 221 & 21,42 & 357,84 & 54,07 & 7,27 & 38,66 & & & & & & & \\
\hline 222 & 26,35 & 285,14 & 60,19 & 5,14 & 34,68 & & & & & & & \\
\hline 225 & 23,41 & 364,96 & 59,65 & 6,09 & 34,26 & & & & & & & \\
\hline 227 & 27,08 & 479,77 & 44,83 & 5,25 & 49,92 & & & & & & & \\
\hline 228 & 26,49 & 571,49 & & & & 50,00 & 200,00 & $43,18 \%$ & $56,82 \%$ & $24,16 \%$ & 881,19 & $29,13 \%$ \\
\hline 229 & 25,24 & 876,88 & 54,36 & 5,79 & 39,86 & & & & & & & \\
\hline 230 & 28,86 & 784,98 & 34,11 & 7,91 & 57,98 & & & & & & & \\
\hline 231 & 25,52 & 529,89 & 43,02 & 4,77 & 52,22 & & & & & & & \\
\hline 233 & 21,97 & 260,99 & 54,92 & 5,01 & 40,07 & & & & & & & \\
\hline 235 & 24,86 & 248,19 & 59,20 & 7,02 & 33,78 & & & & & & & \\
\hline 236 & 24,53 & 272,96 & 74,08 & 7,62 & 18,30 & & & & & & & \\
\hline 237 & 25,73 & 269,28 & 58,94 & 7,44 & 33,62 & & & & & & & \\
\hline 240 & 25,34 & 213,68 & 74,56 & 8,50 & 16,94 & & & & & & & \\
\hline 242 & 23,88 & 138,75 & 54,10 & 7,67 & 38,23 & & & & & & & \\
\hline 243 & 25,64 & 342,44 & & & & 60,00 & 250,00 & $57,93 \%$ & $42,07 \%$ & $23,59 \%$ & 440,99 & $28,82 \%$ \\
\hline 244 & 24,4 & 152,2 & 62,08 & 6,87 & 31,06 & & & & & & & \\
\hline 247 & 25,25 & 391,35 & 54,03 & 5,96 & 40,01 & & & & & & & \\
\hline 248 & 22,88 & 272,15 & 52,06 & 8,22 & 39,72 & & & & & & & \\
\hline 258 & 28,08 & 385,15 & & & & 40,00 & 200,00 & $51,31 \%$ & $48,69 \%$ & $26,45 \%$ & 617,51 & $28,61 \%$ \\
\hline 263 & 28,34 & 447 & 50,13 & 5,57 & 44,30 & & & & & & & \\
\hline 264 & 31,8 & 223,94 & 34,62 & 10,90 & 54,48 & & & & & & & \\
\hline 266 & 32,38 & 654,05 & & & & 40,00 & 220,00 & $69,45 \%$ & $30,55 \%$ & $28,70 \%$ & 210,91 & $28,96 \%$ \\
\hline 270 & 30,6 & 354,04 & 47,81 & 5,59 & 46,60 & & & & & & & \\
\hline 272 & 29,93 & 408,31 & 48,13 & 5,59 & 46,27 & & & & & & & \\
\hline 276 & 29,47 & 1198,55 & 51,41 & 5,97 & 42,63 & & & & & & & \\
\hline 277 & 29,32 & 1561,97 & & & & 70,00 & 350,00 & $49,83 \%$ & $50,17 \%$ & $26,67 \%$ & 661,40 & $24,17 \%$ \\
\hline 287 & 26,52 & 4,3 & 62,72 & 37,28 & 0,00 & & & & & & & \\
\hline 293 & 21,98 & 1,03 & 86,59 & 13,41 & 0,00 & & & & & & & \\
\hline
\end{tabular}

